\title{
Wesen und Wirkungen des Lichts bei Bonaventura
}

Farben sind nur im Licht sichtbar, in der Dunkelheit sehen wir keine Farben - es sei denn an einem phosphoriszierenden Körper, der in Photolumineszenz selbst Licht ausstrahlt. Das legt nahe, sich dem Phänomen des Lichts zu widmen, ohne welches Farben nicht verstanden werden können. Wenn hier eine bedeutsame mittelalterliche Theorie des Lichts ${ }^{1}$ ins Licht der Aufmerksamkeit gestellt und hoffentlich ein Stück weit erhellt und klar wird, ist damit schon die mindestens zweideutige oder zweistufige Begriffsbedeutung angesprochen, die spätestens seit dem Höhlengleichnis in Platons Politeia die Philosophie begleitet: ${ }^{2}$ Das Licht, einerseits sinnlich wahrnehmbares Phänomen und Grundlage der Farbigkeit, wurde andererseits zur Bezeichnung der Möglichkeit von Erkenntnis (Licht, das Einsicht ermöglicht) ${ }^{3}$ wie auch als Metapher für religiöse Erfahrungen (Erleuchtung, Licht des Glaubens) verwendet. Schließlich ist nach 1 Joh. 1,5 (Deus lux est) Gott selbst oberstes Licht. ${ }^{4}$ Im Mittelalter hat Bonaventura diese vielschichtige Begrifflichkeit gerne verwendet und besonders intensiv bearbeitet, wobei er sich durchaus auch für die physischen Phänomene des Leuchtens, Reflektierens und der Farbe interessierte.

Erleichtert wird das Verständnis zunächst durch seine Verwendung von lux in metaphysischer Bedeutung und von lumen für das physische Licht, welches in einem Medium strahlt (radius) und an und um Körpern Glanz (splendor, fulgor) verursacht ${ }^{5}$ und effektives Prinzip des Akzidens Farbe ist. ${ }^{6}$

1 Für Guardini ist Bonaventura ,ihr bedeutendster Vertreter“ (79) und „der Lichtmetaphysiker schlechthin unter den Scholastikern" (83).

2 Sehr nüchtern analysiert Thomas von Aquin in S.Th. I q 67 al die Ausweitung des mit dem Begriff lux Angesprochenen vom Ermöglichungsgrund des Sehens mit dem Gesichtssinn zur Wahrnehmung überhaupt und dann zum geistigen Einsehen (einschließlich des Gott-Sehens). Vgl. Bonaventuras Komm. In II Sent. dist. XIII a1 q1 ad 3; p. 313.

Aus Bonaventuras Kommentar zu dieser dreizehnten Distinctio des II. Sentenzenbuchs wird am häufigsten zitiert, anderweitig nicht spezifizierte Stellenangaben beziehen sich darauf.

3 Bonaventura: „omnis cognitio spiritualis fit ratione lucis“" (In I Sent. dist. III p.1), ,lumen intellec$t u s^{\text {c }}$ (z. B. In II Sent. dist. XXXIX a2 q2). Auf seine Illuminationstheorie, die auch Guardini als im Bildhaften verbleibend kritisiert (Systembildende Elemente; 29), wird hier nicht weiter eingegangen.

4 Z. B. In I Sent. dist. 3 p. 1.

5 In II Sent. dist. XIII a3 q1; p. 325 gibt eine präzise Bestimmung von lumen et radius als qualitas sowie eine sekundäre Definition: radius aër illuminatus secundum diametrum, et lumen aër illu- 
Die relevanten Texte des Sentenzenkommentars stehen in einem spezifischen Kontext, nämlich der Erklärung des Sechstagewerks gemäß Gen. 1. Vom Anliegen einer die Rationabilität der Heiligen Schrift verteidigenden Auslegung her verstehen sich manche Detailfragen und Antwortversuche, die heute weniger systematisches Interesse finden können, z. B. nach der Möglichkeit der Einteilung der Tage vor der erst am vierten Tag erschaffenen Sonne (ein Problem, dem sich Bonaventura mehr als andere Scholastiker widmet), nach dem Beginn aller zeitlichen Abfolge mit der Morgen- oder Abenddämmerung und deren Erklärung (vgl. dub. I; p. 330) oder die zugrundeliegende problematische Annahme des Petrus Lombardus, daß vor der Erschaffung des Lichts, welches erklärtermaßen die erste Unterscheidung der Schöpfung machte, zwar weder Tag noch Nacht, wohl aber Zeit existiert habe. ${ }^{7} \mathrm{Im}$ Hintergrund steht die Dreiteilung des Schöpfungswerkes in opus creationis (nach Gen. 1,1), opus distinctionis (vor allem Gen. 1,4-13) und opus ornatus (ab Gen. 1,14, also 4.-6. Tag). In jedem Fall charakterisiert es die Denkweise Bonaventuras, daß eine nicht von der Theologie erleuchtete philosophische Reflexion zur fruchtlosen oder gar hochmütigen Neugierde verkomme, wohingegen im Rahmen theologischer Leitwissenschaft alle Argumente, von griechischen und lateinischen Vätern wie vom Philosophus und seinen auch arabischen Auslegern eine sinnvolle Funktion erfüllen. ${ }^{8}$

\section{Licht als Urform alles Körperlichen}

Bekanntlich hat der christlich modifizierte Neuplatonismus das Denken des Doctor seraphicus am stärksten geprägt, wonach Licht etwas Substantielles ist, das mit dem Sein und dem Guten konvertibel ist. " „Das Sein selbst ist ein Lichtsein und das Wirken eine Lichtwirkung. ${ }^{10}$ Teilhabe am Sein bedeutet Teilhabe am Licht, was in Analogie

minatus secundum profundum. A 2 q 2 definiert: fulgor, qui est circa corpus luminosum, qui consequitur existentiam lucis in tali materia, et qui etiam est sensus obiectum et operationis instrumentum.

6 lux est de compositione coloris ... tanquam principium effectivum (In II Sent. dist. XIII a2 q2 ad2; p. 321).

7 ante lucem nec dies fuit nec nox, licet tempus fuerit (Liber II sent. dist. XIII cap. II). Anders Thomas von Aquin: Auch die Erschaffung der Engelsnatur und der ungeformten Materie ging der Formgebung nicht zeitlich voraus, sondern der Natur nach. Et sicut natura praecedunt formationem, ita etiam et motum et tempus. (S.Th. I q66 a4)

8 Vgl. einfuihrend zu Bonaventura einschlägige Philosophiegeschichten, z. B. Heinzmann: Philosophie; 223-232.

9 Vgl. Eriugena im Gefolge des maßgeblichen Lichtmetaphysikers Dionysius Pseudo-Areopagita: omnia quae sunt, lumina sunt (Expos. super ierarchiam cael. I,1 in MPL 122; 128). Zur Geschichte der Lichtmetaphysik siehe immer noch den historischen Teil in Baeumker: Witelo; eine Zusammenfassung der Quellen Bonaventuras bei Guardini: Systembildende Elemente; 79-84.

10 Guardini: Systembildende Elemente; 23. 
zur Abstufung sichtbaren Lichts dann auch graduell abgestuft verstanden wird gemäß größerer oder geringerer Nähe zur Lichtquelle. Diese strahlt aus ${ }^{11}$ und wird je verschieden rezipiert und auch weitergegeben, womit die Metapher des Lichtes einen universalen Zusammenhang alles Seienden und seine Einheit vom Ursprung her nahelegt und veranschaulicht.

Vor diesem Hintergund bot sich eine Möglichkeit, dem ersten Schöpfungsbericht mit seiner Unterscheidung des fundamentalen Schöpfungsakts von den folgenden Distinktionen und zunehmend konkreteren Fortbestimmungen sowie weiteren Ausgestaltungen, Ausformungen bis hin zur Schönheit (formositas) einen metaphysischen Sinn abzugewinnen: Nachdem gemäß dem ersten Satz des Buches Genesis Gott Himmel und Erde erschaffen hatte, war eine materia prima anzunehmen, deren Charakter, inanis et vacua zu sein als Formlosigkeit interpretiert wurde. ${ }^{12}$ Durch die folgende Erschaffung des Lichts habe diese eine erste oder fundamentale, allgemeine Informierung erfahren durch die allgemeine Lichtform. ${ }^{13}$

So versteht Bonaventura lux als forma communis, welche die Materie überhaupt informiert oder vorformt; er spricht auch von einer gemeinsamen Natur aller, d. h. himmlischer und irdischer Körper. ${ }^{14}$ Insofern damit zugleich ein Streben nach je spezifischer Ausformung mitgegeben ist, wird verständlicher, daß die gemeinsame Urform des Lichts zugleich als Unterscheidungsprinzip dienen soll, nachdem zu diesem generale principium distinguendi in corporibus dann weitere substantielle Formen hinzutreten, um jedes zu dem zu machen, was es ist. Das ontologische Urlicht ist etwas Formales, da es höchst aktiv ist, wie man aus Erfahrungen mit dem sichtbaren Licht und seinen Farbwirkungen sowie aus Theorien über dessen Wirkungen auf die Elemente in der Tiefe der Erde, auf die Meere, Pflanzen usw. schloß. ${ }^{15}$ Soweit übernimmt Bonaventura die aristotelische Begrifflichkeit, daß Wirkkraft eben der Form zugehört. Anders als im thomasischen Aristotelismus wird in Bezug auf das principium formale davon ausgegangen, daß die Information der Materie eine doppelte sei. ${ }^{16}$ Um die nobelste allgemeine Form gegenüber den etwas zu etwas Bestimm-

11 lux simul est et lucet et illuminat (Bonaventura In II Sent. dist. XIII a1 q2 ad 4; p. 316).

12 Petri Lombardi Liber II Sent. dist. XIII cap. I.

13 Entsprechend lautet die Überschrift von Bonaventuras Kommentar zur XIII. dist. De generali informatione materiae per formam communem lucis.

14 Lux est natura communis reperta in omnibus corporibus tam caelestibus quam terrestribus (dist. XII a2 q1 arg. 4); quod omnia corpora naturam lucis participent (dist. XIII a2 q2 concl.; p. 321); siehe ebd. „divisio textus“. Licht als ,forma prima corporalis" wurde von Robert Grosseteste ausformuliert: De luce seu de inchoatione formarum (Philos. Werke des R. Grosseteste, ed. L. Baur) 1912; 10f. und 51 ff.; englisch „On light“ (Transl. Claire C. Riedl), Milwaukee (Marquette Univ. Pr.) 1978.

15 Siehe a2 q2 arg. 4; p. 319 und Gilson; 315.

16 Ebd. 308f. zur Differenz zum thomasischen Formbegriff. Bonaventura meint, duplex est informatio materiae corporalis, quaedam generalis, quaedam specialis - generalis per formam communem omnibus corporalibus, et haec est forma lucis; specialis vero per alias formas (a1 q1 divi- 
tem vollendenden spezifischen Formen nicht als etwas Defizientes erscheinen zu lassen, betont Bonaventura, daß jene nicht eine unvollkommene Anlage sei, sondern das, was jede andere körperliche Form bewahrt und ihr Wirkkraft gibt:

Forma enim lucis cum ponitur in eodem corpore cum alia forma, non ponitur sicut dispositio imperfecta, quae nata sit perfici per ultimam formam, sed ponitur tanquam forma et natura omnis alterius corporalis formae conservativa et dans ei agendi efficaciam. ${ }^{17}$

Dennoch ist diese allgemeine Lichtform nach a1 q1 etwas Körperliches, womit sich Bonaventura auch (in vorsichtiger Weise) gegen die spiritualistische Interpretation des ersten Satzes der Bibel als Erschaffung der Engelsnatur bei Augustinus wendet. Dafür argumentiert er neben Hinweisen auf Autoritäten mit Folgerungen aus einer wörtlichen Genesisexegese, vor allem mit der Körperlichkeit des in Gen 1,1 genannten abyssus, dessen Dunkelheit ebenso etwas Körperliches sein müsse wie das diese vertreibende Licht. Das wichtigste Argument beruht also auf der physikalischen Funktion des Lichts als konstitutivem Teil der erfahrbaren Welt.

Dazu paßt die über Petrus Lombardus übernommene Rede von der leuchtenden Wolke oder dem Lichtschleier (lucida nubes), womit Bonaventura öfters die von der Sonne doch unterschiedene Körperlichkeit umschreibt, ${ }^{18}$ als einer Art Vorform oder früherem status der am vierten Tag aus dem Lichtschleier geformten Sonne. ${ }^{19}$

\section{Spannungen zwischen theologischen Argumentations- interessen und naturphilosophischen Einsichten}

Unter der Annahme, daß die Rede von erstem, zweitem usw. Schöpfungstag wörtlich gemeint sei, schließt sich daran die zweite Frage an, wie dieses Urlicht Tag und Nacht voneinander unterscheiden konnte vor der Erschaffung der Sonne, die dasselbe ja durch

sio textus). Thomas von Aquin kritisiert u. a. dieses Verständnis des Hylemorphismus in S.Th. I q. 66: A1 argumentiert dagegen, daß eine materia zeitlich der Formierung vorausgegangen sein könnte, wie manche Kirchenväter meinten. Dicere igitur materiam praecedere sine forma, est dicere ens actu sine actu: quod implicat contradictionem. Nec etiam potest dici quod habuit aliquam formam communem et postmodum supervenerunt ei formae diversae, quibus sit distincta. Dies richtet sich gegen Bonaventuras Theorie der Vielheit der Formen - was identisch sei mit den Vorstellungen vorsokratischer Naturphilosophen von Feuer, Luft oder Wasser als materia prima. Demnach müßte diese schon substantial bestimmt sein, und folgende (Weiter-)Formungen könnten nur akzidentell sein, womit das Werden zu einem bloßen Wandel erklärt würde.

17 A2 q2 ad 5; p. 321.

18 Petri Lombardi Liber II Sent. dist. XIII cap. II; im Komm. al q1 ad 5; p. 313, al q2 ad 4; p. 316 oder ähnlich a2 q1 ad $6 ;$ p. 318.

19 A1 q1 ad 6; p. 313 und dub. II, p. $330 f$. 
ihren Umlauf zustande bringt. Wofür sich Bonaventura hier interessiert, nenne ich theologische Protokosmologie. ${ }^{20}$ Gegen die mit einem besonderen göttlichen Befehl argumentierenden griechischen Kirchenväter hält Bonaventura es für vernünftiger, weil dem natürlichen Vermögen eher entsprechend, daß das Urlicht Tag und Nacht hervorbrachte durch seine Bewegung - in einer unbeholfen wirkenden Analogie zum Sonnenlauf, ${ }^{21}$ aber nahe am Sentenzentext, der in cap. III die Frage, wo das Licht erschaffen wurde, beantwortet: in illis partibus facta, quas nunc illustrat solis diurna lux und gleich die Tag-Nacht-Unterscheidung motu suo als wahrscheinlich behauptet.

Erhellend ist die Entgegnung auf den zweiten Einwand, der besagt, daß die erste Materie ganz uniform gewesen sein muß, und das Urlicht unbeweglich, weswegen kein Unterschied von Tag und Nacht möglich gewesen sei: Dagegen behauptet Bonaventura einfach, jenes Licht hätte nicht überall leuchten können, so wie die Sonne auch nicht. ${ }^{22}$ Darum hätte die Illumination sukzessive stattgefunden und den Zeitablauf (temporalis successio) verursacht. Die Sinnhaftigkeit dessen sei nicht zu bezweifeln, weil es dem göttlichen Arrangement gefallen habe, die Welt wie einen wunderschönen Gesang durch eine bestimmte Zeitenfolge zu zieren. ${ }^{23}$

Die Frage nach dem Ort des Urlichts (bei Petrus Lombardus cap. III) ist Bonaventura demgegenüber weniger wichtig; er referiert zwei konkurrierende Theorien, nämlich das Aufleuchten im Meridian (wofür Beda genannt wird) und im Osten (nach Hugo von St. Viktor) und stellt am Ende der Quaestio fest, eine Entscheidung sei nicht leicht und mache auch keinen großen Unterschied. Zu bemerken ist, daß beide Hypothesen unbefragt von einer festen Örtlichkeit und terrestrischen Orientierung ausgehen.

So ist eine eigenartige Zwiespältigkeit festzustellen: Einerseits wirkt Bonaventuras Lichtmetaphysik der q1 - jedenfalls für den in moderner Physik nur Allgemeingebildeten - erstaunlich kompatibel mit physikalischen Forschungen des 19. und 20. Jahrhunderts: ${ }^{24}$ In formaler Hinsicht kann die Suche der neuzeitlichen Physik nach einer einheitlichen Theorie der Physis auf Bonaventuras Postulat eines einheitlichen Grundprinzips von allem materiell Seienden bezogen werden, das er im Lichtcharakter alles Seienden erkennt.

20 Zur Kosmologie siehe seinen Komm. zur dist. XIV.

21 rationabilior, quia virtuti naturali possibilior, scilicet quod lux illa noctem et diem faciebat suo motu, sicut sol sua facit actione (a1 q2 concl.; p. 315).

22 materia ex omni parte erat uniformis; dicendum, quod etsi ex omni parte esset influentiam lucis aequaliter nata suscipere, illa tamen lux non poterat undique illuminare, sicut et sol munc non potest (a1 q2 ad 2; p. 316).

23 Divinae autem dispositioni placuit, mundum quasi carmen pulcherrimum quodam decursu temporum venustare (ibid.).

24 Die gelegentlichen Hinweise der Scholiasten von 1885 auf die Lehren der nostra aetate physici wären demnach einer Revision zu unterziehen. Diese erfordert freilich mehr physikalische Fachkenntnis als den vorliegenden Gelegenheitshinweisen bezw. Gedankenanregungen auch im letzten Teil dieses Beitrags zugrunde liegt. 
In inhaltlicher Rücksicht zeigt sich weiters eine Analogie zur Doppelnatur des Lichts als Teilchen und als Welle darin, daß es nach Bonaventura einerseits formalen Charakters ist, andererseits aber körperlich (aliquid corporis), wobei er das Wesentliche eines Körpers in seiner Ausdehnung (extensio) $\operatorname{sieht}^{25}$ und offenbar nicht in dem, was heute Masse heißt.

Licht hat nun in seinem Teilchencharakter doch etwas Materielles an $\operatorname{sich}^{26}$, während alle Materie in ihren kleinsten Bestandteilen etwas Energetisches ist. ${ }^{27}$ In Anbetracht dessen, daß der Doctor seraphicus für die Mittelstellung des Lichts mit sowohl formalen als auch materiellen Eigenschaften vor allem aufgrund seiner Wirkkraft argumentiert, könnte man lux versuchsweise mit Energie übersetzen. Die Lichtartigkeit aller, auch der physischen Wirklichkeit nach Bonaventura scheint zur allmählichen Ausweitung der Korpuskel-Welle-Doppelnatur zu passen, welche seit Louis de Broglies Entdeckung des Wellencharakters auch massebehafteter Teilchen stattfand. Eine Übereinkunft und Diskussionsbasis zeigt sich folglich in der Annahme eines grundlegend dynamischen Charakters der ganzen Wirklichkeit einschließlich aller Körper.

Die moderne Physik scheint mehr die Zusammengehörigkeit von Energie und Materie zu betonen, während Bonaventura sie zunächst als metaphysische Prinzipien der Form und der bloßen Materie auseinanderhält - um aber das Entstehen von allem damit erklären zu können, daß das Licht oder die Energie die erste und fundamentale, konstitutive Information mitteilt.

Eine solche hier angedeutete Gesprächsmöglichkeit ergibt sich weniger daraus, daß Bonaventuras Lichtmetaphysik in Kosmologie und Physik übergeht, sondern daraus, daß die Physik auf metaphysische Fragen stößt. Denn jener Übergang vollzieht sich, wie zu sehen war, aufgrund des Wunsches, ein sehr wörtliches Verständnis der Schöpfungserzählung zu plausibilisieren. Und darin besteht das Andererseits der Zwiespältigkeit: Denn eine fundierende Lichtartigkeit alles Materiellen als solchen, dessen primäre Informierung oder Energetisierung auf dem bloßen, noch nicht spezifizierten Niveau des Vorhanden- und Etwas-Seins bezw. Sein-Könnens (al q1), ist schwerlich vereinbar mit Annahmen wie der Lokalisierung und der zeitlichen Periodisierung dieser Energie zur Tageseinteilung vor Existenz der Gestirne inklusive der Sonne (a1 q2). Die (im Kommentar zur dist. XIV wieder aufgegriffene) Exegese von Gen. 1 schien ihm dies aber zu erfordern. Die ersten Worte der Vulgata, in principio, wurden sehr schnell in zeitlichem Sinne, als Beginn einer sukzessiven Temporalität mißverstanden und damit physisch statt metaphysisch oder auch ontisch statt ontologisch. Die theologische Vorentscheidung fördert eine Unfähigkeit, auf der prinzipiellen Ebene zu bleiben und stört hier die Fruchtbarkeit des metaphysisch-naturphilosophischen Ansatzes.

25 Cum etiam nullum corpus careat extensione, et omnis extensio fundetur super materiam corporalem, nullum corpus potest esse sine materia (a2 q1 concl.; p. 317f.).

26 Der theoretische Grenzbegriff eines unbewegten Photons ohne Ruhemasse fällt hier nicht unter den Begriff des Lichts.

27 Nostra autem aetate non pauci physici docent, naturam vel qualitatem lucidi esse in omni corpore (Scholion p. 322). 


\section{Vom Licht als Urform zur wahrnehmbaren Leuchtkraft}

Der zweite Artikel widmet sich dem Wesen des Lichts, seiner natura in se. Für die Meinung Licht sei ein Körper, wird vor allem Augustinus angeführt, für die Hypothese, es sei die natürliche Eigenschaft des Feuers, Johannes Damascenus, und für die Formhaftigkeit Aristoteles, denn das Wirkprinzip sei Form, und Licht sei maxime activa, insofern substantialis forma. ${ }^{28}$ Bonaventura hält fest: Reine Form ohne alle Materie ist nur Gott; andererseits kann Licht kein Körper sein, denn es vervielfältige sich aus sich selbst heraus, ${ }^{29}$ was Körpern unmöglich ist.

Wenngleich die ganze Erläuterung der dist. XIII vom körperlichen Licht handelt, ist es doch nicht einfach als Körper zu verstehen, sondern als Form oder auch Qualität eines leuchtenden Körpers - jedenfalls in abstrakter Redeweise als das, was dem Körper Leucht- und Wirkkraft gibt. Indessen meint $l u x$ bisweilen konkret eine leuchtende Substanz - so sei Augustinus zu lesen. ${ }^{30}$,Wir können demnach unter Licht eine die körperliche Materie aktualisierende Form verstehen, die nur in unserm Denken für sich existiert, wenn wir sie durch Abstraktion von ihrer Materie loslösen." (Gilson; 307) Durch seine maximale Aktivität und Kraft, sich selbst zu vervielfältigen und zu verbreiten nimmt es eine Mittelstellung zwischen den rein geistigen Formen und den konkreten körperlichen Formen ein. ${ }^{31}$ Das entspricht dem Bestreben des Doctor Seraphicus, den gesamten Kosmos als differenzierte, in sich verbundene organische Einheit zu deuten, die vermittels des Lichts mit dem reinen Licht des göttlichen Urprinzips verbunden ist. ${ }^{32}$

Damit argumentiert Bonaventura auf der prinzipiellen, metaphysischen Ebene, die mit dem exegetischen Anliegen der vorigen Quaestio nicht leicht zu harmonisieren ist. Das zeigt sich wiederum in der eigenartigen Diskussion des sechsten Einwands, der sich ja aus der Beantwortung der vorigen Frage ergibt, daß jenes zuerst erschaffene Licht durch seine Bewegung Tag und Nacht bewirkte, also ein Körper gewesen sein müsse: „Licht heißt dort die kleine Leuchtwolke, die wahrlich ein Körper war; und sie war nicht geschaffen [im strengen Sinn des Wortes], weil aus der präexistenten Materie die Form des Lichts auf göttliche Weise eingedrückt oder herausgeführt wurde. Aus dem

28 In II Sent. dist. XIII a2 q2 ad 6; p. 322.

29 Cum lucis sit ex se ipsa se ipsam multiplicare (a2 q1 concl.; p. 318).

30 Lux potest dici in abstractione, et sic nominat formam corporis luminosi, per quam corpus illud habet lucere et agere; et sic dicit Damascenus, quod lux est qualitas ignis. Aliquando nominat in concretione ipsam, scilicet substantiam luminosam... Et per hunc modum loquitur Augustinus (ibid.).

31 Inter ceteras formas corporales est maxime activa et quasi medium tenens inter formas spirituales et corporales (In II Sent. dist. XIV a1 q3 2 conc1.).

32 Lux inter omnia corporalia maxime assimilatur luci aeternae (a2 q2; p. 319). 
Vorigen ist also klar, daß Licht, in eigentlicher und abstrakter Redeweise, kein Körper ist, sondern die Form eines Körpers. ${ }^{\text {(33 }}$

Wegen des zeitlichen und ontologischen Vorrangs und wegen der Fähigkeit, substantiale Formen zu erwirken, ist jedoch nach a2 q2 lux forma substantialis corporum, d. h. allgemeines metaphysisches Prinzip, das in abgestufter Weise partizipiert wird als Sein. Den Argumenten dafür, daß Licht nur eine akzidentelle Form sei, gesteht er eine teilweise Plausibilität zu, insofern das die wahrnehmbare Wirkung an den Seienden der selbst nicht wahrnehmbaren substantialen Form sei - eben Glanz und Farbe. Diese sind Wirkungen des inkorporierten Lichts - akzidentelle und sichtbare Eigenschaften. Bonaventura faßt zusammen:

lux dupliciter potest accipi. Uno modo lux dicitur ipsa forma, quae dat esse corpori lucido, et a qua luminosum corpus principaliter est activum, sicut a primo movente et regulante. Alio modo lux dicitur ipse fulgor, qui est circa corpus luminosum, qui consequitur existentiam lucis in tali materia, et qui etiam est sensus obiectum et operationis instrumentum; et sic est accidentale complementum. ${ }^{34}$

Die zweite Hinsicht ist weniger problematisch als die erste - diese führte zur Problematik der pluralitas formarum, welche hier nicht aufzugreifen ist, wo es letztlich um die Erklärung der eben akzidentellen Lichtwirkungen geht. Auffällig ist, daß das Licht in ontologischer Bedeutung hier als Form und Bewegkraft (nur) der leuchtenden Körper bezeichnet wird, während es sonst als allgemeine Form aller Körper gedeutet wird (vgl. Anm. 14). Auch die Lösung des zweiten Zweifels, was im Fortgang des Schöpfungswerks aus dem Urlicht wurde, daß daraus alle leuchtenden Himmelskörper gemacht wurden (dub. II; p. 331), restringiert den universalen metaphysischen Ansatz auf die supralunare Sphäre. Insoweit anzunehmen ist, daß diese Einschränkung vom unmittelbaren Diskussionskontext der Quaestio bedingt ist und Bonaventura an der Allgemeinheit der Erstinformation aller körperlich Seienden durch das Licht oder die Energie festhält, wäre eine Erklärung darüber zu ergänzen, wieso bestimmte Arten von Körpern die substantiale Grundform durch Leuchten akzidentell sichtbar werden lassen, im Gegensatz zu anderen, nicht lumineszierenden Körpern. ${ }^{35}$

33 Lux ibi vocatur mubecula lucida, quae vere corpus erat; et illa non fuit creata, pro eo quod fuit ex praeexistenti materia forma lucis sibi divinitus impressa vel educta. Sic igitur ex praedictis patet, quod lux, proprie et abstracte loquendo, non est corpus, sed forma corporis (In II Sent. dist. XIII a2 q1 ad 6; p. 318).

34 A2 q2 concl.; p. 321.

35 Einen „phänomenologischen“ Hinweis in diese Richtung gibt die Beobachtung, daß es kaum einen opaken Körper gebe, der nicht durch Bearbeitung zum Glänzen gebracht werden kann: $E t$ quod omnia corpora naturam lucis participent, hoc satis de plano ostendunt, quia vix est corpus opacum, quin per multam tersionem et politionem possit effici luminosum, sicut patet, cum cinere fit vitrum, et de terra carbunculus (a2 q2 concl.; p. 321). 
Im dritten Artikel werden Wirkung und Ausstrahlung des Lichts - lumen - weiter ausgeführt. Die erste Frage ist, ob das von einem leuchtenden Körper emittierte Licht selbst körperlich sei. Ein Gegenargument, welches sich ähnlich bei Thomas von Aquin findet, ${ }^{36}$ ergibt sich daraus, daß kein Körper subito bewegt werden könne, während man ja die Ausbreitung des Lichts in instanti beobachte. Interessanterweise macht sich Bonaventura selbst den Einwand, das könne ja auch sehr schnell geschehen - doch daß dies tatsächlich mit nicht wahrnehmbarer, aber endlicher Geschwindigkeit sein könne, war ihm, der das mit der Schallgeschwindigkeit vergleicht, nicht vorstellbar. ${ }^{37}$ Andere Argumente gegen die Körperlichkeit führen die Fähigkeit des Lichts an, sich in alle Richtungen zugleich auszubreiten (opp. 5), seine unvermischte Gleichzeitigkeit (und Gleichörtlichkeit) mit der Luft (opp. 6), sein kontinuierliches Werden aus der emittierenden Lichtquelle heraus, ${ }^{38}$ und daß jeder Körper entweder aus etwas (verursachendem oder aufnehmendem Körper) oder aus nichts (nur aus Gott) entstehen müsse, was beides für lumen nicht zutrifft (opp. 8). Aus diesen Gründen sei jene Interpretation am vernünftigsten, die das sichtbare Licht (lumen) präzise als Eigenschaft des Lichts (lux) und folglich nicht als Körper oder dessen Wirkung auffaßt - während genau dies akzeptabel erscheint, insofern mit radius und lumen die erleuchtete Luft gemeint wird (concl.; p. 325). Denn Licht brauche immer ein geeignetes Medium als Leiter. Für dieses hat es nicht die Rolle der Form, kann sich mit der Materie nicht zu einer Substanz verbinden, ist aber aufgrund seiner Wirkkraft doch formähnlich.

Die wahrnehmbaren Phänomene des Rückprallens oder Abweisens von Licht an einem opaken Körper (zweites Argument für die Körperlichkeit) sind dann mit dessen Unfähigkeit, die (Vervielfältigungs-)Kraft des Lichts aufzunehmen, zu erklären. Diese kehre dann zurück, was auch Spiegelungen verständlich mache; der aufnehmende Körper muß irgendwelche bestimmten Qualitäten haben, ohne welche das Licht nicht in ihm oder durch ihn wirken kann. ${ }^{39}$ Die Erklärung von Kreuzungen und Brechungen des Lichts bemüht konsequenterweise ebenso Eigenschaften der die Strahlen tragenden Luft (intersecatio et confractio radiorum, ibid. ad 3 ).

Angesichts des auch heute nicht aufgelösten Paradoxons, daß Licht sowohl als Wellenfunktion wie als körperliches Teilchen $\mathrm{zu}$ definieren ist, erscheint es durchaus als

36 S.Th. I q 67 a2.

37 A3 q1 opp. 3 und ähnlich opp. 4; p. 324; vorsichtiger in I. Sent. dist. XXXVII pars II a2 q3 ad1. Vgl. a3 q1 ad 1; p. 325: Das Scheinen ist nicht als Ortsbewegung, sondern als Entstehung und Ausbreitung von Licht zu verstehen.

38 lumen in aëre habet suum esse in fieri, quia continue egreditur a sole (a3 q1 opp. 7; p. 324). Vgl. die Diskussion der ersten, Seneca zugeschriebenen Position, die einen Verbrauch des Sonnenkörpers erklären können müßte, was mit der angenommenen Inkorruptibilität der Himmelskörper unvereinbar ist (concl.; p. 325).

39 Ibid. ad 2.; p. 325 f. und ibid. ad 5: cum lumen sit forma corporalis, indiget medio deferente. 
eine Leistung, wie Bonaventura seine Theorie von der Unkörperlichkeit des physischen Lichts (mit Zusatzhypothesen) an die einzigartigen Lichtphänomene anzupassen wußte.

Dasselbe Bemühen um Phänomengerechtigkeit sowie um Vermittlung divergierender Autoritäten, um die una veritas integra (p. 328) zu finden, charakterisiert seine Lösung der zweiten Frage, ob lumen eine substantiale oder akzidentelle Qualität sei. Energie an sich ist ihm ja substantielle Form; das Licht in einem Medium wie der Luft ist eine nicht wahrnehmbare, aktive Kraft, wodurch ein leuchtender Körper auf niedrigere Körper wirkt und insofern substantiell - allerdings in der Weise eines Akzidens. Insofern damit indessen eine sichtbare Qualität gemeint ist, sei es eine besondere, nicht naturale und nicht von der Substanz untrennbare Art von Akzidens. Sein Verhältnis zum Medium ist anders als das zu einem ersten Subjekt zu verstehen, nämlich als Habitus.

\section{Attraktivität und Problematik der Energietheorie}

Im Allgemeinen unterscheidet Bonaventura klar Licht oder Energie an sich, Licht als wahrnehmbares Leuchten eines Mediums und als Farberscheinung: Lux potest tripliciter considerari, scil. in se et in transparenti et in extremitate perspicui terminati; primo modo est lux, secundo modo lumen, tertio modo hypostasis coloris. ${ }^{40}$ Doch die Konfusion der ersten beiden beim Bemühen, das Sechstagewerk zu erklären, verunklärt oder verdunkelt den ontologisch interessanten Ansatz und stört die Klärung des Zusammenhangs oder Übergangs von der Metaphysik zur Physik, von lux zu lumen.

Das zeigt eine überraschende Parallele zu einem Grundmuster heute beliebter Lichtoder Energiebegriffe - sowohl im metaphysischen Ansatz wie auch in der problematischen Durchführung:

Das Prinzip esoterischer Weltbilder ist ein „feinstoffliches“ universales Licht oder eine Urenergie (wobei nicht leicht ersichtlich ist, inwieweit das materiell, inwieweit geistig ist), woraus emanierend die ganze Wirklichkeit gebildet wird in einem ununterbrochenen Fluß - was oft recht vage mit Theorieelementen der gegenwärtigen Physik begründet wird. ${ }^{41}$ Das erscheint im Prinzip vergleichbar mit Bonaventuras systembildenden Lehren vom Licht, von der influentia und dem neuplatonischen Grundmuster von exitus der Wirklichkeit aus dem Urprinzip und reditus zurück. Der Doctor Seraphicus würde wohl gegen die esoterische Energielehre sagen, daß das kosmisch-körperliche Licht seine tatsächlich universale Wirkkraft nur vom reinen, unkörperlichen Licht, der transzendenten absoluten Form und Wirklichkeit her habe und nur im Rahmen der

40 In I Sent. dist. XVII pars. 1 q1 corp.; p. 294.

41 Siehe Runggaldier: Philosophie der Esoterik 36 ff., der diese „typisch monistische Ereignisontologie“ (36) erstmals philosophisch bearbeitete. Das könnte als Ausgangsbasis für eine Diskussion auch mit dem von Runggaldier nicht erwähnten Bonaventura dienen. 
Hierarchie zu verstehen sei, was einen energetischen Monismus und Pantheismus ausschließt. ${ }^{42}$ Energie, die uns am auffälligsten als Licht erscheint, ist nicht reine Form, was nur von Gott gesagt werden kann, obwohl es aufgrund seiner feststellbaren Wirkkraft etwas Formales an sich hat. Es ist aber als Wirkendes Teil der physikalischen Welt, und zwar etwas für die materielle Wirklichkeit Konstitutives, also körperlich, ohne ein reiner, ausgedehnter Körper zu sein. So wenig sich bei Bonaventura ein Dualismus von materieller und geistiger Welt findet, der gerade vom Nachdenken über das Phänomen des Lichts verhindert wird, so wenig kann er als Gewährsmann für eine monistische Wirklichkeitsinterpretation dienen. Das wäre, wie gezeigt, klarer ohne seinen vorschnellen Übergang zur Protokosmologie des ersten Schöpfungstags. Was bei Bonaventura ein theologisch motiviertes Anwendungsproblem ist, könnte im esoterischen Weltbild ein grundsätzliches Problem sein. Beide beziehen ihre Attraktivität aus einer nicht analytischen, sondern tendentiell holistischen Denkform.

Guardini warnt am Ende seiner Habilitationsschrift über Bonaventura vor den Grenzen der von ihm - allerdings nicht ausschließlich - angewandten ,organischen Methode": Sie ,ist vielleicht für das religiöse Leben befruchtender und vermag eher dem ganzen Menschen etwas zu geben; dafür ist sie aber beständig einer Gefahr der Vermengung mit gefühls- und phantasiemäßigen Elementen ausgesetzt, überschreitet leicht die methodische Grenze, verwechselt Bilder mit Argumenten, praktische Brauchbarkeit mit logischer Richtigkeit, und Gemütswerte mit sachlicher Wahrheit.“ (220) Die Lichttheorie Bonaventuras erscheint so zwar als belastet durch ein allzu spezielles bibelhermeneutisches Interesse und insofern kritisierbar, aber als naturphilosophisch und physikalisch interessant, als spirituell anregend und in dieser Hinsicht als ein attraktiver kritischer Gesprächspartner für heute.

Insoweit es möglich ist, lux mit Energie wiederzugeben, hätten Bonaventura und die moderne Physik darin ein gemeinsames Prinzip der Physis - ein Prinzip ihrer Einheit. Probleme ergeben sich mit dem Prinzip oder den Prinzipien der Vielfalt der Seienden:

Die resolutive physikalische These, daß Materie im Kleinsten eine Form von Energie und beides grundsätzlich konvertibel ist, erklärt zwar Phänomene der Umwandlung von Materie in Energie (wie es in der Sonne laufend geschieht), aber die Entstehung von Materie aus Energie, insbesondere das Werden der Vielfalt der Formen, bleibt ein Problem, das womöglich auf physikalischer Ebene gar nicht lösbar ist. Die Physik will das auch kaum, während die Esoterik hier weitergehende Erklärungsansprüche zu haben scheint.

Bonaventuras Lichtmetaphysik setzt demgegenüber ein rein ideelles, metaphysisches Prinzip für die Vielfalt konkreter Ausformungen an - letztlich den Schöpferwillen, der in einer absoluten Metapher identisch ist mit dem Licht an sich, dem reinen Licht oder

42 Vgl. a2 q1 ad 4; p. 318 zu lux spiritualis - lux corporalis und Guardini: Systembildende Elemente; $217 \mathrm{f}$. 
der Quelle allen Lichts. Der Übergang jedoch zur physischen Energie, die meßbare materielle Wirkungen bis hin zu den sichtbaren Farben hat, der Übergang von lux zu lumen, erscheint als zu wenig geklärt. Der Rückgang oder Rückschluß von Phänomenen des lumen zum Prinzip lux ist hauptsächlich getragen von den eben nicht körperlichen Fähigkeiten des Lichts.

Als Gewinn dieses Ansatzes jedoch bleibt, daß der Kosmos von seinem Ursprung her als eine dynamische Einheit verstehbar wird - und daß Farbe, Licht in seiner Sichtbarkeit, als äußerste Erscheinungsform dessen begriffen wird, was allem Sein gibt und damit der Glanz eines Höchstprinzips ist. 


\section{Literaturverzeichnis}

S. Bonaventurae Opera omnia: In secundum librum sententiarum, Ad Claras Aquas (Quaracchi) 1885.

The Works of Bonaventure, Cardinal, Seraphic Doctor, and Saint. Translated from the Latin by J. de Vinck; 5 vol., Paterson N.J. 1960-66.

Baeumker, Clemens: Witelo: ein Philosoph und Naturforscher des XIII. Jahrhunderts, Münster (Aschendorff) $1991^{2}$.

Gilson, Etienne: Die Philosophie des heiligen Bonaventura, Köln (Hegner) $1960^{2}$.

Guardini, Romano: Systembildende Elemente in der Theologie Bonaventuras: Die Lehren vom lumen mentis, von der gradatio entium und der influentia sensus et motus, Hg. W. Dettloff, Leiden (Brill) 1964.

Heinzmann, Richard: Philosophie des Mittelalters, Stuttgart (Kohlhammer) $1998^{2}$.

Runggaldier, Edmund: Philosophie der Esoterik, Stuttgart (Kohlhammer) 1996. 
